Original Research Paper

\title{
A Qualitative Assessment of VANET Routing Protocols Under Different Mobility Models
}

\author{
Meriem Houmer and Moulay Lahcen Hasnaoui \\ Information and Communication Systems Engineering Research Team, \\ Mathematical Modeling and Computer Science Laboratory, \\ National Graduate School of Arts and Crafts, Moulay-Ismail University, Meknes, Morocco
}

Article history

Received: 8-10-2018

Revised: $17-12-2018$

Accepted: 22-01-2019

Corresponding Author:

Meriem Houmer

Information and

Communication Systems

Engineering Research Team,

Mathematical Modeling and

Computer Science Laboratory,

National Graduate School of

Arts and Crafts, Moulay-Ismail

University, Meknes, Morocco

Email: houmer.m@gmail.com

\begin{abstract}
Vehicular Ad-hoc Networks (VANET) is an advanced wireless network that came to increase traffic safety, efficiency and to improve driving experience. The high mobility of nodes is the major characteristic in these networks. The aim of this paper is to figure out the impact of various mobility models on the performance of Ad-hoc On-demand Distance Vector (AODV), Cluster-Based Directional Routing (CBDR) and Greedy Perimeter Stateless Routing (GPSR) protocols. In this study, we analyze the performance of these routing protocols in different mobility models namely: Street Random Waypoint (STRWP), Gauss Markov and Freeway having varying speed. The simulation results show that STRWP provides a good performance with the three routing protocols in terms of packet delivery ratio, throughput and end to end delay.
\end{abstract}

Keywords: VANET, High Mobility, AODV, CBDR, GPRS, STRWP, Gauss Markov, Freeway

\section{Introduction}

Vehicular Ad-hoc Networks (VANET) is an autonomous and self-organizing wireless communication network, where vehicles act as mobile nodes, to supply communications amid vehicles, also between vehicles and interfaces named Roadside Unit (RSU) (Houmer et al., 2018). VANET have distinctive characteristics like distributed processing, the massive number of nodes, the raised speed of nodes, the topology of network is greatly variable, the transmissions of the signal is blocked by buildings and the frequent partition of the network (Martinez et al., 2008).

Therefore, ad-hoc routing protocols must adapt to its specific features, for having the exact performance measurements in VANET.

Mobility is the main restraint faced in the vehicular networks, for that reason the performance investigations of vehicular network routing requisite the accurate prediction of nodes mobility and this is achieved by the great choice of the mobility model.

The aim of this paper is to presents the performance analysis of three mobility models which are Street Random Waypoint, Gauss Markov and Freeway on AODV, GPSR and CBDR Routing protocols in VANET environment with respect to various metrics that are packet delivery ratio, throughput and end-to-end delay.
This paper is structured as follows: First of all, we present VANET routing protocols. Next, we introduce the different mobility models utilized in the simulation. After that, we detail metrics used to evaluate the routing protocols in the mobility models. Then, we present the simulation and analysis. Finally, we conclude with a conclusion.

\section{Vehicular Ad-Hoc Network Routing Protocols}

Routing is a wide concept utilized in mobile and vehicular ad-hoc network. Several routing protocols has been designing for communication in ad-hoc environment. The high mobility of nodes in VANET makes routing a very hard task in this network.

VANET routing protocols are classified to: Topologybased routing protocol, cluster-based routing protocol, position-based routing protocol, geo-cast routing protocol and broadcast routing protocol (Kumar and Dave, 2011). Our study mainly focuses on the three first categories.

\section{Topology-Based Routing Protocol}

These protocols utilize the existing links information in the network in order to transfer packets to the destination. 


\section{Ad-hoc On-Demand Distance Vector (AODV)}

AODV routing protocol is a topology-based routing protocol that routes data across wireless mesh networks. It is a routing protocol that gives roads on demand, that's mean it builds routes between nodes when needed. It is able to unicast and multicast routing. It is loop-less, starts automatically and hosts a large number of mobile nodes. For multicast groups, AODV builds a tree structure. This routing protocol does not consume a lot of energy and does not require significant computing power. Therefore, it can simply be integrated on small mobile devices. All nodes in this protocol maintain a routing table to store information regarding active routes from source to destination. The table contains the number of hops, next hop, destination sequence number, active neighbors for a route and the destination of a route table entry and its time of expiry.

To find route between source and destination, the first node broadcast a Route Request message (RREQ) to all the neighbors which pursue to send the same message to their neighbors, till the destination is attained. All nodes record the sequence number and broadcast-id of the node sending the data in their routing table conducive to preserve the information of the most recent route and the loop-free If an intermediate node receives the same copy of the request, it rejects it directly without routing it to the destination (Khairnar and Kotecha, 2013).

When the RREQ message attains the destination or an intermediate node with an available route to the destination, a Route Reply (RREP) message is sends back to the sender. Then a route is built between nodes. When the establishment of the route becomes invalid, a link rupture is signaled to the source via a Route Error (RERR) message (Kumar and Dave, 2011).

\section{Cluster-Based Routing Protocol}

In this category, a group of nodes are identifying as part of a cluster and a node designated as cluster head will broadcast the packet into the cluster. A virtual infrastructure of the network shall be founded in this protocol by clustering nodes to provide scalability.

\section{Cluster-Based Directional Routing (CBDR)}

CBDR forms clusters from vehicles traveling in the same direction. These vehicles are capable to communicate with its neighboring cluster through radio communication.

The cluster head broadcasts a LEADER packet to its neighbors containing its location and grid coordinates. If there is a Roadside Unit (RSU) in the area it will become the cluster header. Whether a node does not receive LEADER packet, it broadcast APPLY packet. If the cluster head of this cluster receives the APPLY packet, it responds to this packet by sending another APPLY. Otherwise, if the node does not receive any APPLY packet from the cluster head or other node after a broadcast delay, it considers itself as the new cluster head. Wherever the cluster head leaves the geographic, it broadcasts a LEAVE packet containing its location in the grid. Another node stores this packet until the selection of a new cluster head. The new cluster head uses this information for routing the data (Satyajeet et al., 2016).

In routing procedure, firstly, the source enquires the cluster head if the destination is in the same cluster. If in, it sends data directly to the destination, else it forwards a Route Request (RREQ) packet to the cluster head. Then this cluster head chooses a forwarding cluster head. The next forwarding cluster head informs if the destination is in its cluster. If in, the forwarding cluster head sends RREQ packet to the destination. When the destination node receives RREQ packet, it will reply to the forwarding cluster head with a Route Reply (RREP) due to the reverse routing table, then a link from the forwarding cluster head to the destination is established (Song et al., 2010). The link will maintain only if there is one cluster head in an intermediate cluster.

This protocol contemplates speed and direction which are significant parameters in VANET, unlike CBR which does not consider them.

\section{Position-based Routing Protocol}

Position-based routing protocols use geographic placing information to choose the following forwarding hops. Therefore, no global route between source and destination nodes needs to be established.

\section{Greedy Perimeter Stateless Routing}

Greedy Perimeter Stateless Routing (GPSR) is one of the best protocols of position-based routing. It uses greedy forwarding to transmit packets to neighboring nodes closest to the destination. This method is also known as greedy forwarding.

Each node systematically broadcasts a beacon message to all its neighbors containing its id and position. If any node does not receive a neighbor's beacon message for a specific delay, the GPSR router suppose that the neighbor has failed or out of range and removes it from its table. It makes greedy forwarding decisions using information about the immediate neighbors of the network. For any node, if a greedy transmission is not possible, it uses the perimeter of the regional strategy to find the next transmission hop (Kakarla et al., 2011).

GPSR consists of two methods for transmit packets: Greedy forwarding and perimeter forwarding.

\section{Greedy Forwarding}

Is used to forward data to the nodes closest to the destination. In particular, if a node knows the positions of its closest neighbors, the locally optimal choice of the next hop is the geographically nearest neighbor of the destination (Fig. 1). So, the retransmission successively follows closer geographical jumps until the destination is reached (Alsaqour et al., 2015). 


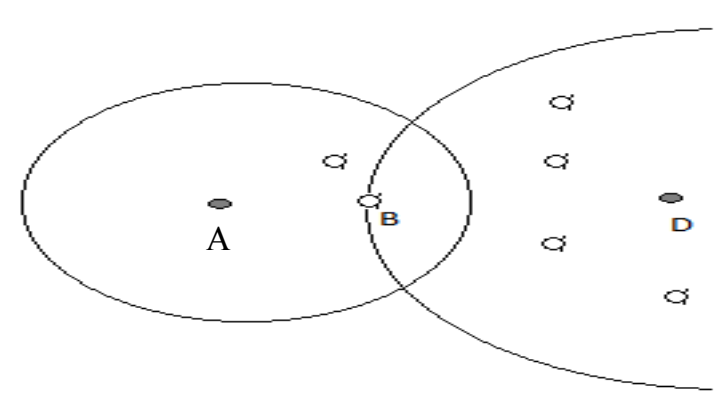

Fig. 1: Greedy forwarding mode

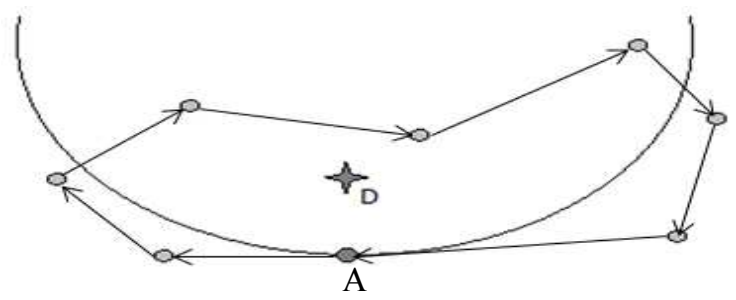

Fig. 2: Perimeter forwarding mode

\section{Perimeter Forwarding}

Is used in areas where there is no near node to the destination. This algorithm uses the right-hand rule, which is defined as follows: Arriving at node $x$ by node $y$, the next traversed edge is the next sequentially counterclockwise around $\mathrm{x}$ from the edge $(x ; y)$.

The state accumulated in these packets is cached by nodes, which restore local maximal to an edge advance by rotating to a node at a perimeter closer to the destination (Fig. 2). This approach requires an uncrossed heuristic to force the rule of the right hand to find the perimeters surrounding the voids in the regions where the edges of the graph intersect (Karp and Kung, 2000).

\section{Mobility Models}

Various mobility models are suggested in order to evaluate and analyze the performance of a vehicular adhoc network protocol.

\section{Street Random Waypoint Mobility Model}

STRWP is a mobility model for VANET, that use a realistic vehicular traffic model for nodes circulating in a real street map data. In an urban grid, STRWP presents a car-following interaction between vehicles, traffic control and stop signs at road intersections.

The concept of STRWP mobility model and that of Random Waypoint are similar. However, street random waypoint model defines at each junction a vehicle trajectory. In fact, each vehicle has a specified probability to change the direction at a junction independently (Mota et al., 2014).
Initially, vehicles are placed randomly in a lane of the map which each vehicle is assigned a speed equal 0 (vehicles are stopped). For each section of a road, STRWP preserves some information like the road class, the start and end points of the section and the name of the street (Amici et al., 2014).

\section{Freeway Mobility Model}

The simulation area of Freeway Model is depicted by a generated map, which contains several freeways compound of many lanes for traffic in both directions. However, mobile node subsequent a specific direction, thus each vehicle in this model are limited to their lanes on freeways. There are no intersections in this model because vehicles circulate in highways.

Initially, vehicles are randomly deposed in the lanes. Speed (S) of each vehicle is temporally dependent on its precedent speed.

Formally:

$$
\left|S_{i}(t+1)\right|=\left|S_{i}(t)\right|+\operatorname{random}()^{*}\left|a_{i}(t)\right|
$$

A Safety Distance (SD) must be maintained between two subsequent nodes moving in the same lane.

While two Vehicles on the same lane with a Safety Distance, the speed of the second vehicle must not surpass the speed of the first vehicle, so the second one decelerates and lets the first vehicle forward away (Divecha et al., 2007).

Formally: if $i$ is before $j$ in its freeway lane:

$\forall i, \forall j, \forall t$, Distance $_{i j}(t)<S D \Rightarrow\left|S_{j}(t)\right|<\left|S_{i}(t)\right|$

From the above Equations (1 and 2), the Freeway mobility model is anticipated to have high temporal and spatial dependence. It sets strict geographic restrictions on vehicles movement. Since, vehicles move in the freeway lane until arriving the limit of the simulation area. This scenario is definitely unrealistic (Rupinder and Gurpreet, 2014).

\section{Gauss-Markov Mobility Model}

The Gauss Markov model represents a broad scale of user mobility models. This system depends on correlated velocity mobility patterns. The prediction is made available to mobiles nodes (vehicles) and network. Therefore, the vehicle is conscious of its position prediction in time. Furthermore, a vehicle verifies regularly its location and update it when it achieves a threshold farness to the predicted direction.

Initially, vehicles are placed randomly in the grid. For the movement of each vehicle a mean Speed (S) and mean Direction (D) are affected. In a specific time, vehicles compute their movement based on their previous speed and direction with a degree of randomness assimilated in the calculation (Liang and Haas, 2002). 
The vehicles are supposed to move in a period of time $(\mathrm{t})$ with a computed speed and direction in a time gap. This speed and direction are calculated in the following formula:

$$
\begin{aligned}
& S_{t}=\alpha S_{t-1}+(1-\alpha) \bar{S}+\sqrt{\left(1-\alpha^{2}\right) S_{t-1}^{G}} \\
& D_{t}=\alpha D_{t-1}+(1-\alpha) \bar{D}+\sqrt{\left(1-\alpha^{2}\right) D_{t-1}^{G}}
\end{aligned}
$$

where, $S_{t}$ is the vehicle's speed at time $t$ and $D_{t}$ is the direction. The parameter $\alpha$ is bounded between 0 and 1 $(0<\alpha<1)$. It is employed to merge the degree of randomness in the moment of computing speed and direction of vehicles movement for a time gap. $\bar{S}$ and $\bar{D}$ are the asymptotic mean of $S_{t}$ and $D_{t}$ when $t$ approaches infinity. $S_{t-1}^{G}$ and $D_{t-1}^{G}$ are an independent, uncorrelated and stationary Gaussian process, with mean $\mu_{x}=0$ and standard deviation $\sigma_{x}=$ $\sigma$, where $\sigma$ is the asymptotic standard deviation of $S_{t}$ and $D_{t}$ when $\mathrm{n}$ approaches infinity.

In a specific time gap, the next position is computed depending on the current position of the vehicle. Specifically, at the moment $(\mathrm{t})$, a vehicle location is given by the following equations:

$$
\begin{aligned}
& X_{t}=X_{t-1}+S_{t-1} \cos D_{t-1} \\
& Y_{t}=Y_{t-1}+S_{t-1} \sin D_{t-1}
\end{aligned}
$$

where, $\left(X_{t}, Y_{t}\right)$ and $\left(X_{t-1}, Y_{t-1}\right)$ are the $X$ and $Y$ coordinates of vehicle's positionat $(t)$ and $(t-1)$ time gap and $S_{t-1}$ and $D_{t-1}$ are the speed and direction of vehicle at $(t)$ and $(t-1)$ time gap (Katkar and Ghorpade, 2016).

\section{Performance Metrics}

Metrics help to analyze and measure the performance of routing protocols under the different mobility models. We are focused in this paper on three performance metrics which are:

\section{Packet Delivery Ratio}

Packet delivery ratio (PDR) is defined as the ratio of packets received by the destination to those sent from the CBR source. Thus, a raised packet delivery ratio means that the routing protocol is reliable and efficient. Mathematically, it can be shown as:

$$
P D R=\frac{1}{c} \times \sum_{1}^{n} \frac{\text { Packet received }}{\text { Packet sent }}
$$

where, $c$ represents the number of connections between vehicles.

\section{Throughput}

Throughput is defined as the quantity of packet transmitted from a vehicle to another per unit time. It is calculated in Kbps. Throughput comprises frequent topology changes, limited power, restricted bandwidth and faithless communication. A lofty throughput network is demanded. Mathematically, it is represented as:

Through put $=\frac{\text { Pck size }}{\text { Sp time }- \text { St time }} \times \frac{8}{100}$

where, $P c k$ in the size of packets received, $S p$ is the simulation stop time and $S t$ is the simulation start time

\section{End-to-End Delay}

The delay of a packet is the time consumed to move from the source to the destination through the network. likewise, End-to-End delay is the average time between the emission of a packet and its reception divided by the number of packets received. the best performance is done with a lower End-to-End delay. Mathematically, is defined as:

$$
\text { End to End delay }=\sum_{1}^{n} \frac{\text { Delay }}{\text { packets recieved }}
$$

\section{Simulation-Based Analysis}

In this section, we produce the results of the effect of the different mobility models represented in the previous section on AODV, CBDR, GPSR routing protocols. This simulation is performed on a vehicular ad-hoc network by varying vehicle's speed.

Firstly, we present the simulation tools also the parameters of the simulation then we analyze the results.

\section{Simulation Tools}

\section{Network Simulator}

Network Simulator 2 (NS2) is a free software for discrete event simulation widely used in academic research and industry. However, it is utilized for studying the dynamic nature of communication networks. NS2 admits the simulation of different routing protocols and functionality of the wireless and wired network as well as ad-hoc network.

NS2 allows users to simulate their corresponding behaviors by choosing the topology of the network, routing and multicast protocols, transport (TCP, UDP, RTP, SRM), the application (Web, FTP, Telnet...) and many others over wireless and wired networks.

NS2 code is written in C++ and OTCL interpreter, thus generating an output file for NAM (Network animator) (Singh et al., 2012).

\section{Network Animator}

Network Animator (NAM) is a Tcl/TK based animation tool permit to display packet and network simulation traces. It supports topology layout, packet level animation and various data inspection tools. This tool plots the nodes that exchange the information with 
each other in a location presented in the script and display the output (Poonia and Bhargava, 2016).

\section{Simulation of Urban Mobility}

Simulation of Urban Mobility (SUMO) is an open source, highly portable, microscopic and large road traffic simulation created to manipulate the wide road networks.

This simulator is basically created by researchers of the Institute of Transportation System at the German aerospace center. SUMO is certified under the GPL. It authorizes users to constitute a personalized road topology and also, to get various map formats of different cities in the world (Saini and Mahapatra, 2014).

\section{Simulation Parameters}

The parameters used in this simulation are shown in Table 1.

Table 1: Simulation environment

\begin{tabular}{ll}
\hline Parameter & Value \\
\hline Channel type & Wireless \\
Routing Protocol & AODV, CBDR, GPSR \\
Traffic type & CBR \\
Time of Simulation & $1000 \mathrm{~s}$ \\
Queue Length & 2048 Bytes \\
X and Y Dimensions of topography & $500 \times 1500 \mathrm{~m}$ \\
MAC Protocol & $802.11 \mathrm{Ext}$ \\
Number of nodes & 100 \\
Speed & $20,40,60,80,100$, \\
& $120,140 \mathrm{~m} / \mathrm{s}$ \\
\hline
\end{tabular}

\section{Simulation Results}

In our simulation, number of vehicles are constant but speed of each one is varied.

The comparative graphs of the three routing protocols "AODV, GPSR and CBDR" in different mobility models "STRWP, Gauss Markov and Freeway" are obtained in the figures thereafter. This study is in regard to three performance metrics: Packet Delivery Ratio (PDR), Throughput and End-to-End delay.

\section{Packet Delivery Ratio (PDR)}

Figure 3-5 presents the performance of AODV, CBDRP and GPSR routing protocols at different mean speed regarding Packet delivery ratio, over three mobility models i.e., Street Random Waypoint, Gauss Markov and Freeway.

We observe that, for the three mobility models, GPSR performed better than AODV and CBDR. However, if we compared the three routing protocols in the different mobility model, we notice that GPSR attains a higher packet delivery ratio varying between 91 and 51 for freeway model, 66 and 44 for Gauss Markov and 77 and 51 for street random waypoint mobility model. AODV and CBDRP are having the lowest values of PDR. Accordingly, we can say that GPSR is the best routing protocol in term of PDR and freeway is the best mobility model, since it gives better performance (best values) for the three routing protocols.

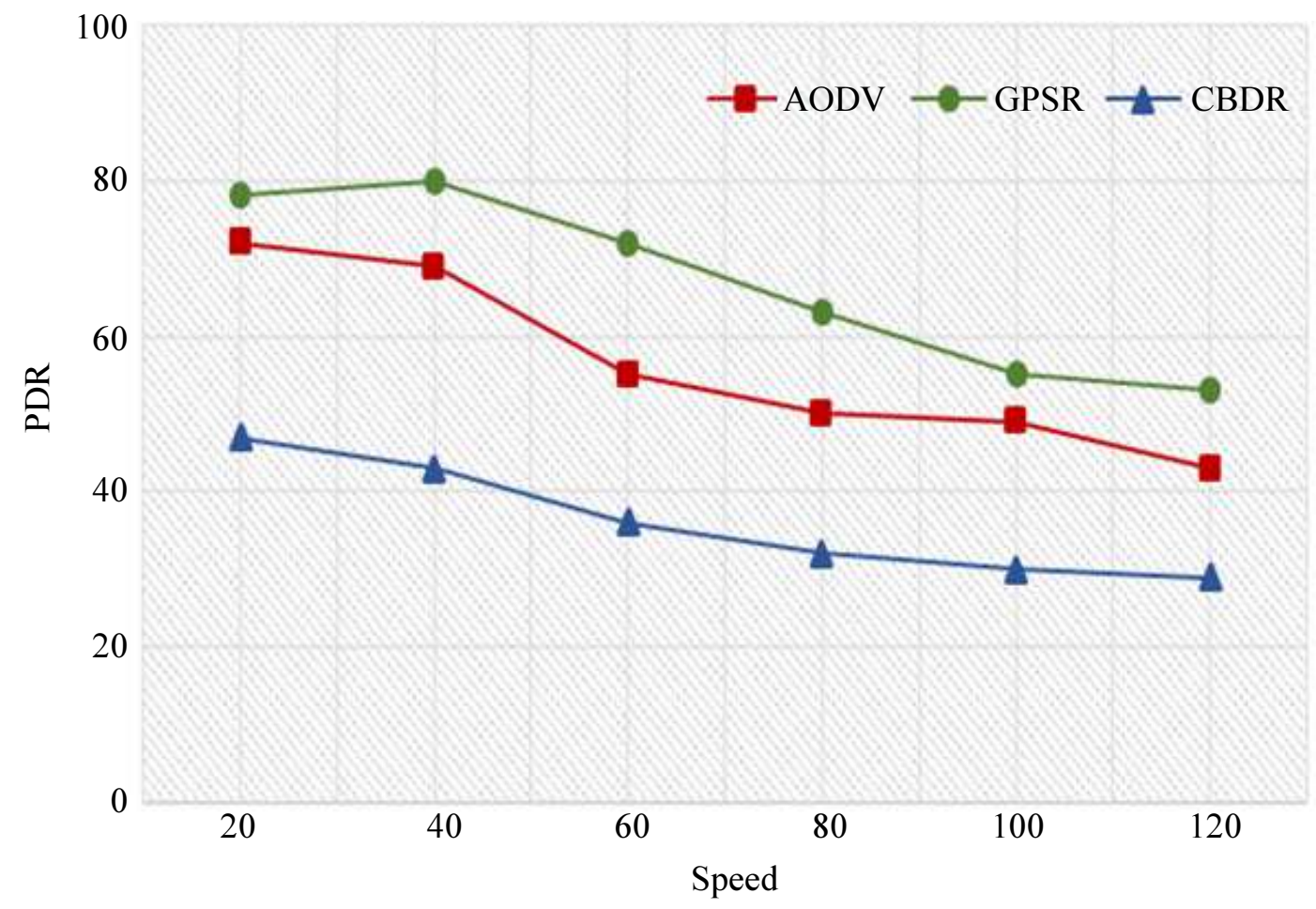

Fig. 3: Variation of PDR over Speed in Street Random Waypoint mobility model 


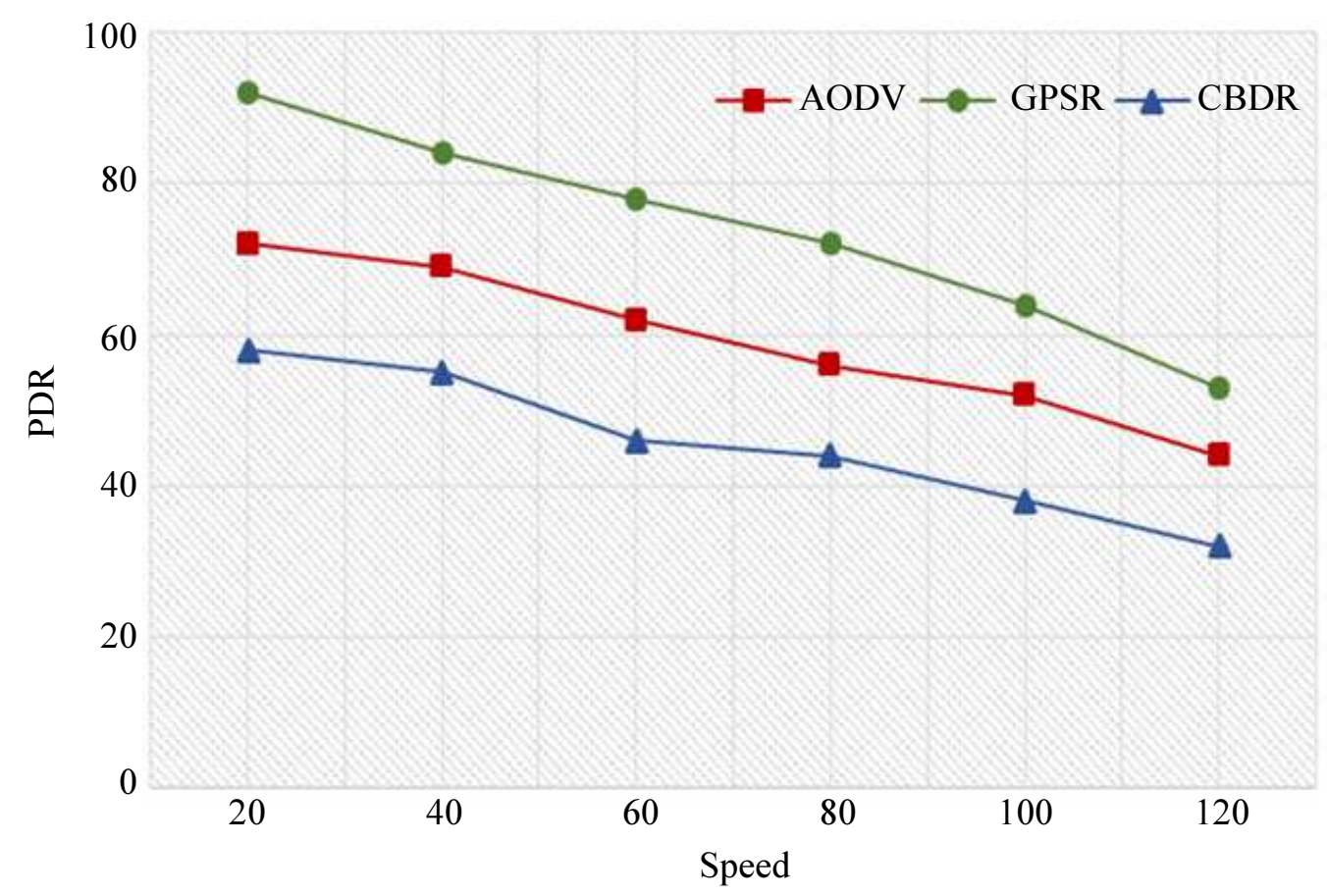

Fig. 4: Variation of PDR over Speed in Freeway mobility model

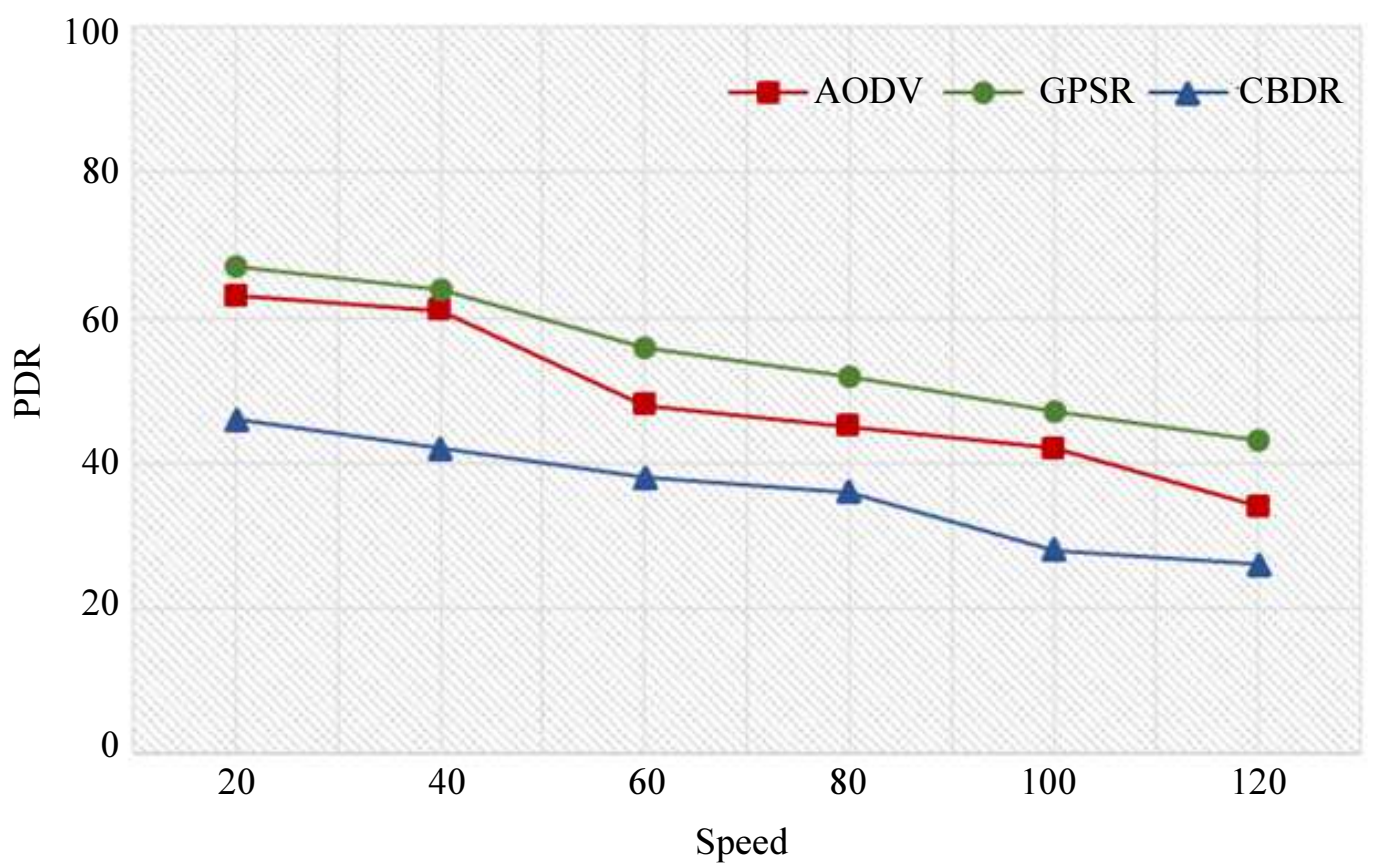

Fig. 5: Variation of PDR over Speed in Gauss-Markov mobility model

\section{Throughput}

Figure 6 to 8 presents the impact of speed on the throughput of AODV, GPSR and CBDR routing protocols for each mobility model. Network size, speed and mobility models have led to increasing the throughput for the three protocols by analyzing throughput GPSR have the best values followed by AODV then CBDR routing protocol.

With increasing speed, we assume that the three routing protocols perform better under freeway and Street Random Waypoint than Gauss-Markov mobility models. 


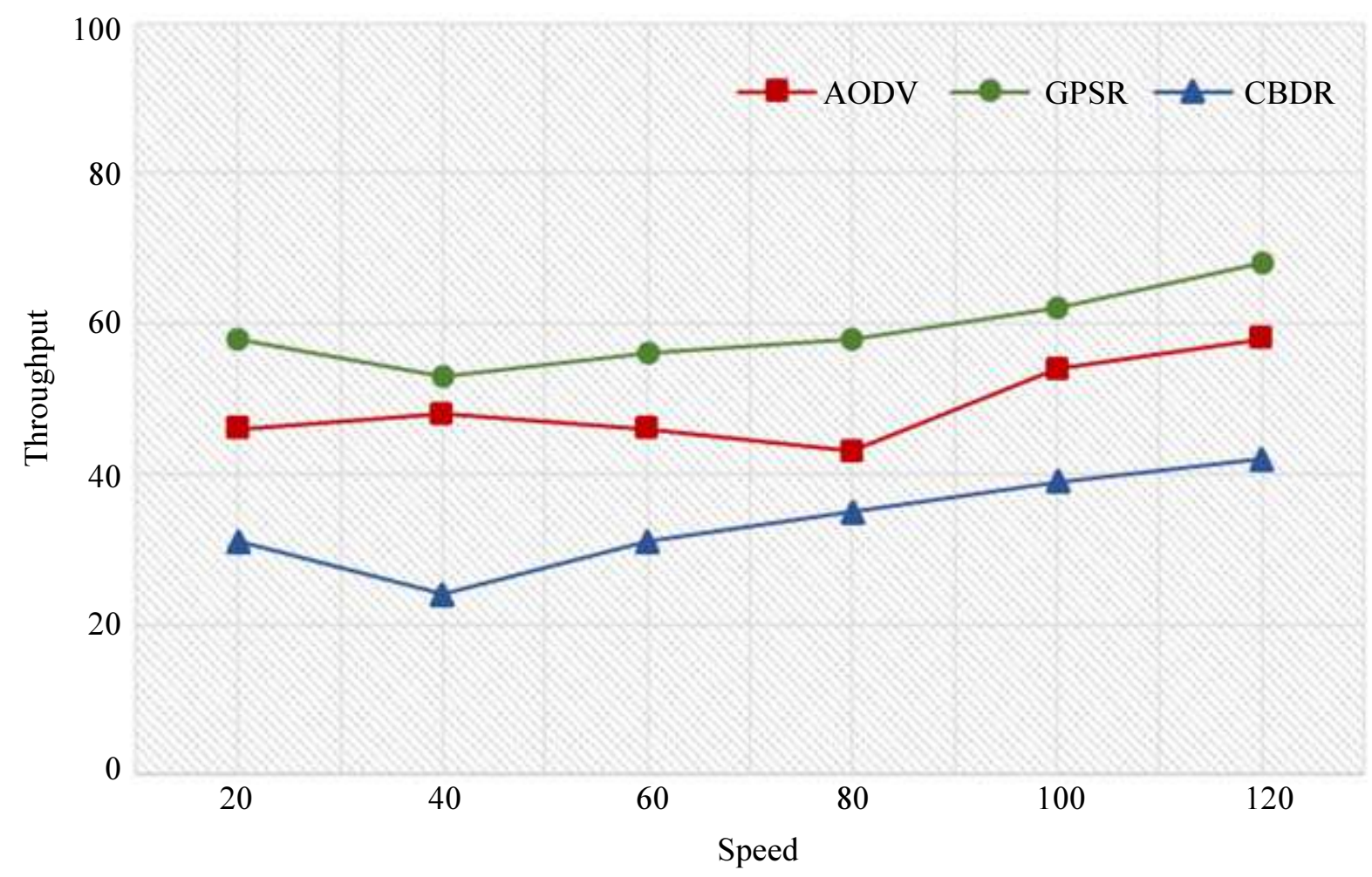

Fig. 6: Variation of Throughput over Speed in Street Random Waypoint mobility model

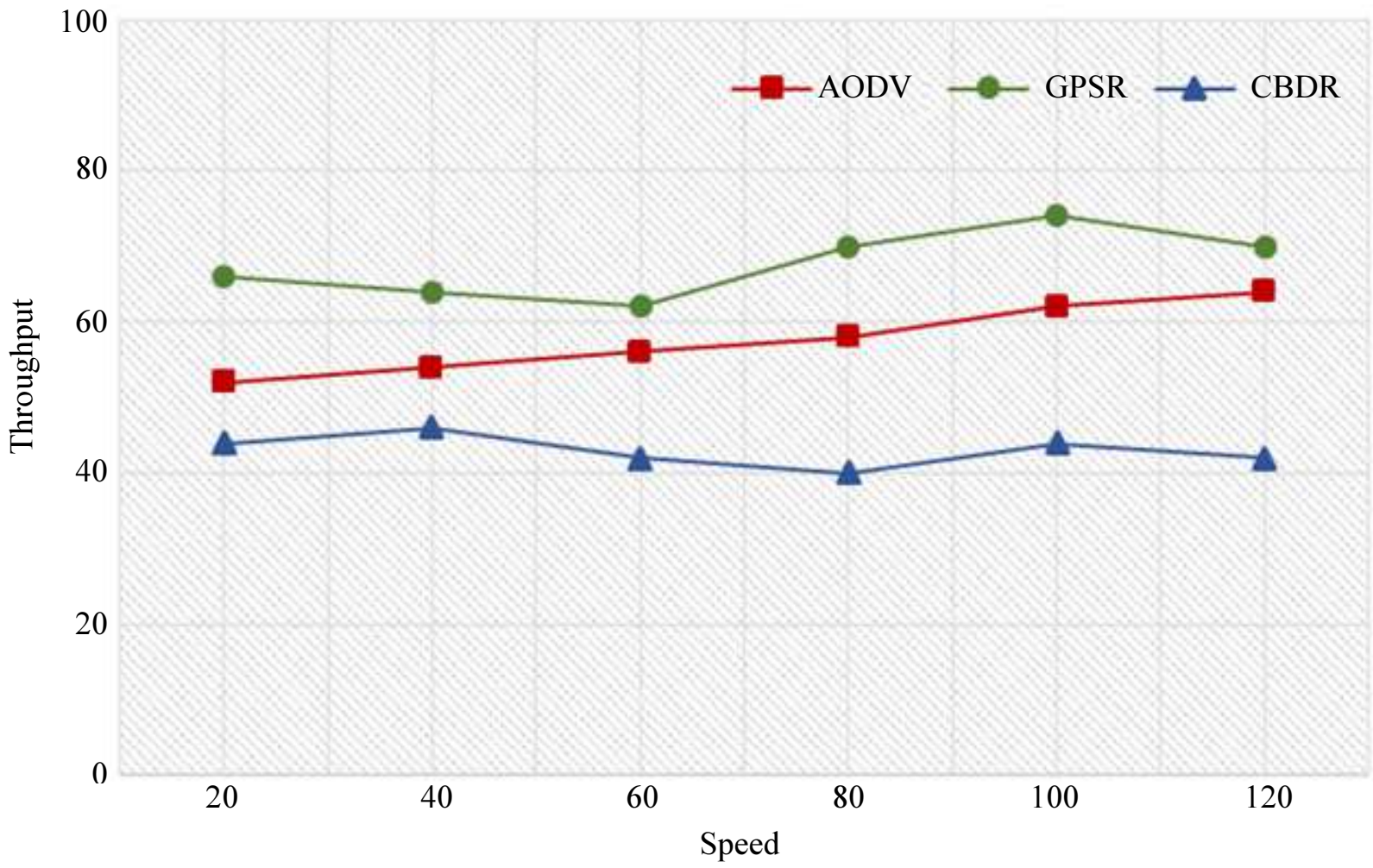

Fig. 7: Variation of Throughput over Speed in freeway mobility model 


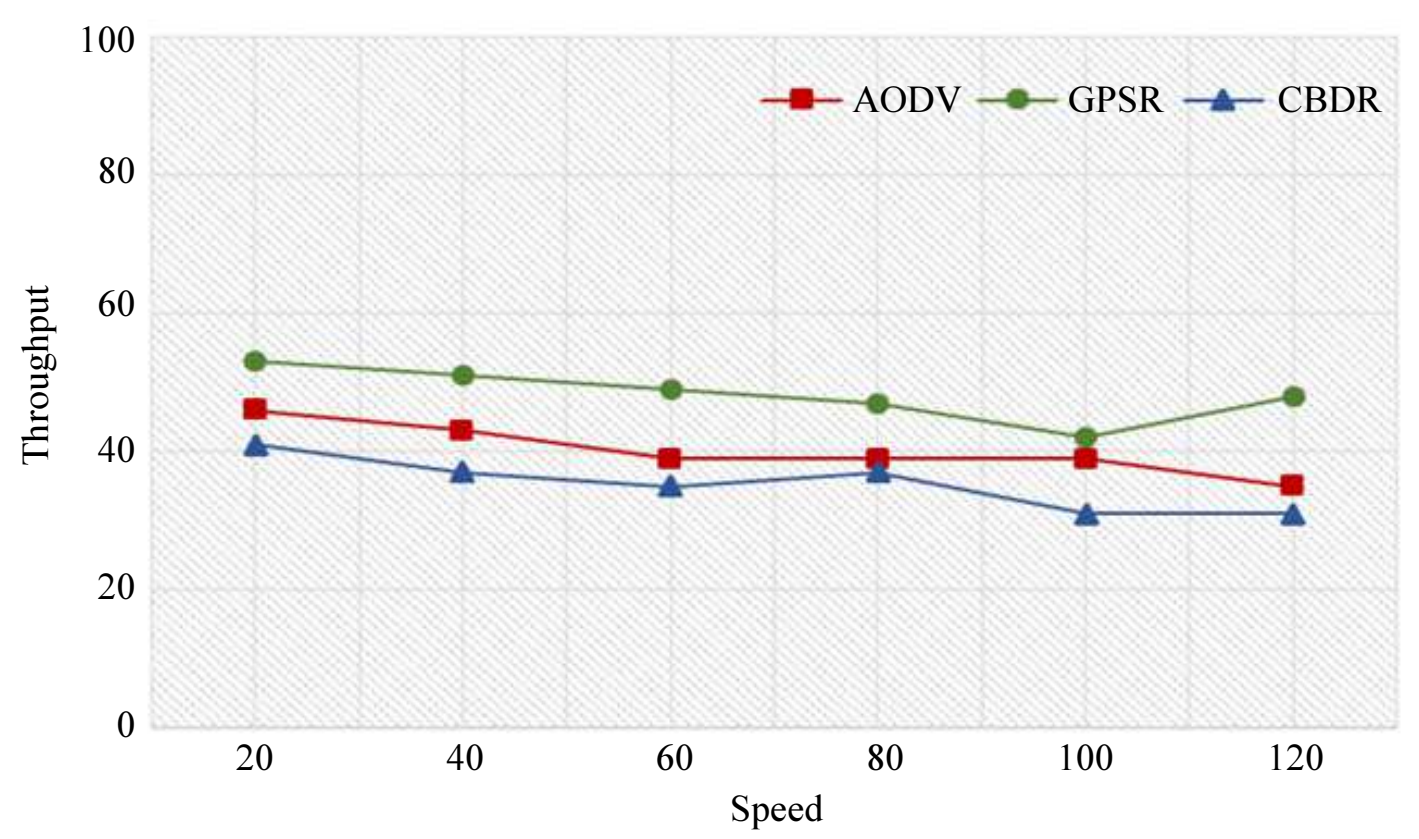

Fig. 8: Variation of Throughput over Speed in Gauss-Markov mobility model

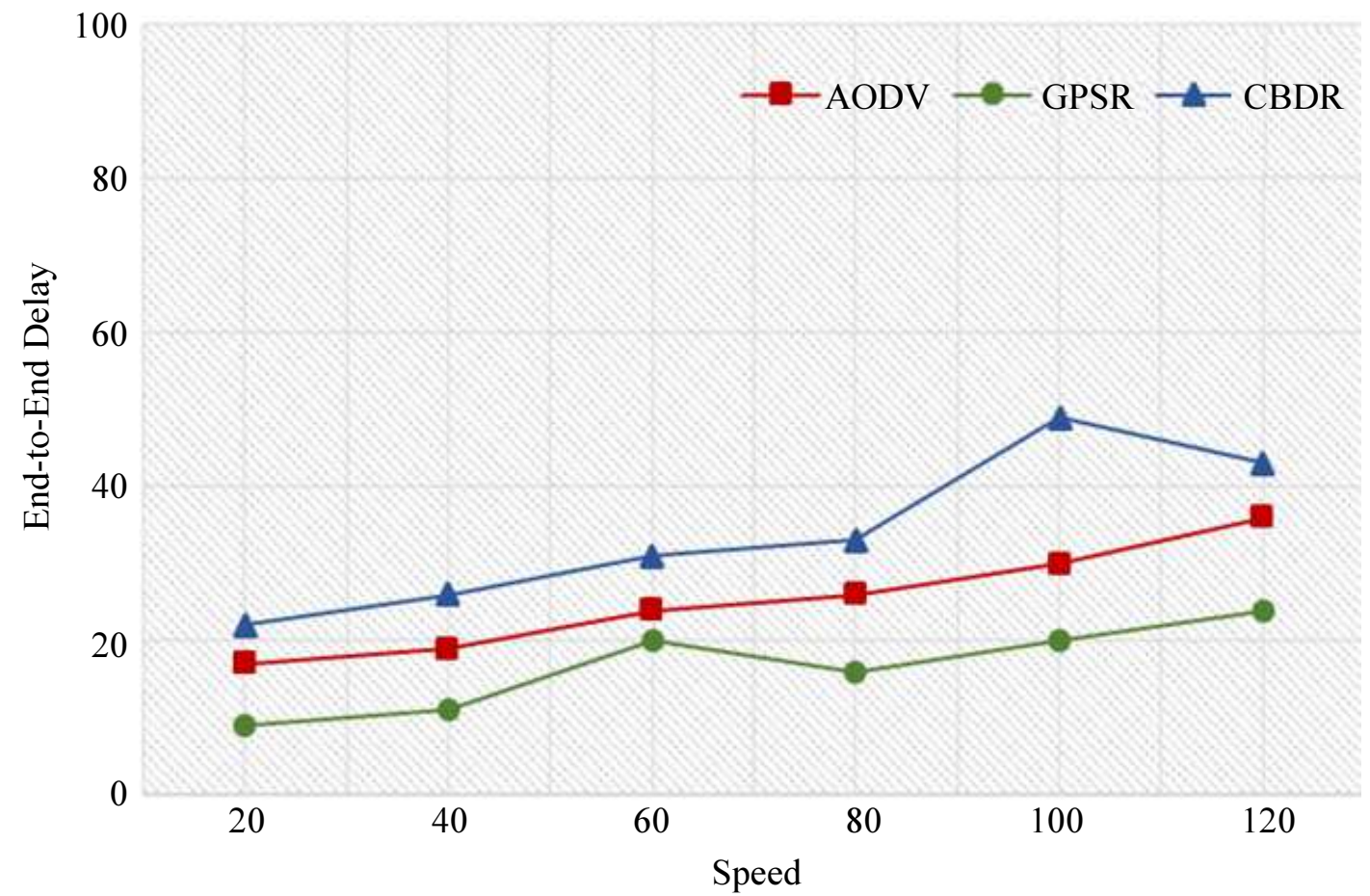

Fig. 9: Variation of End-to-End Delay over Speed in Street Random Waypoint mobility model

\section{End-to-End delay}

Figure 9 to 11 shows the effect of speed on the End-toEnd Delay. As shown in the graphs, GPSR has the lowest End-to-End Delay and CBDR has the highest values.

The graphs clearly show that when speed increase, the end to end delay of the three mobility models raise and routing protocols have lofty values when we use Gauss-Markov mobility model and nethermost values with freeway mobility models.

So, we can say that the freeway model is better than Street Random Waypoint and Gauss Markov mobility models in term of End-to-End Delay and GPSR is the best routing protocol in the same metric. 


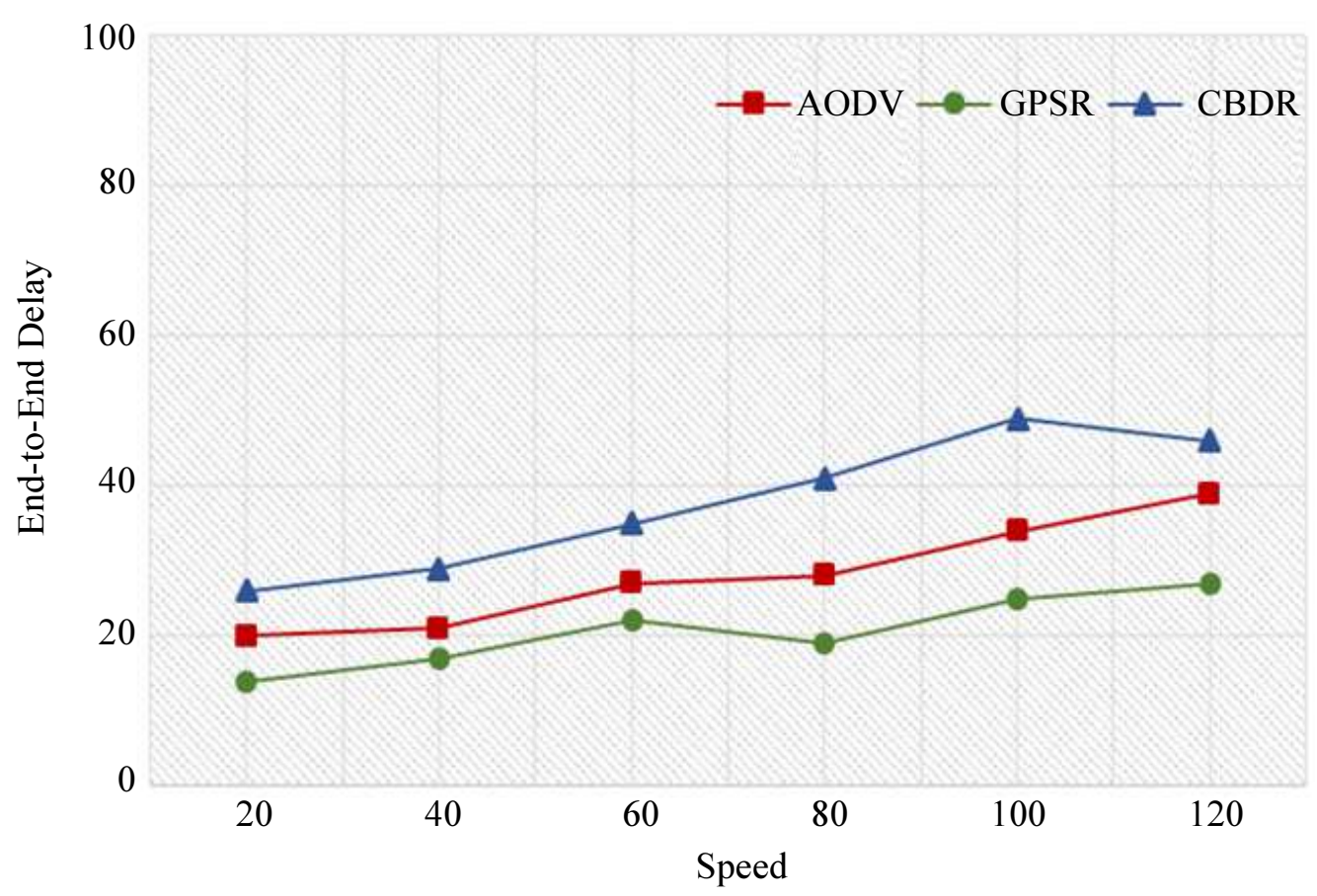

Fig. 10: Variation of End-to-End Delay over Speed in freeway mobility model

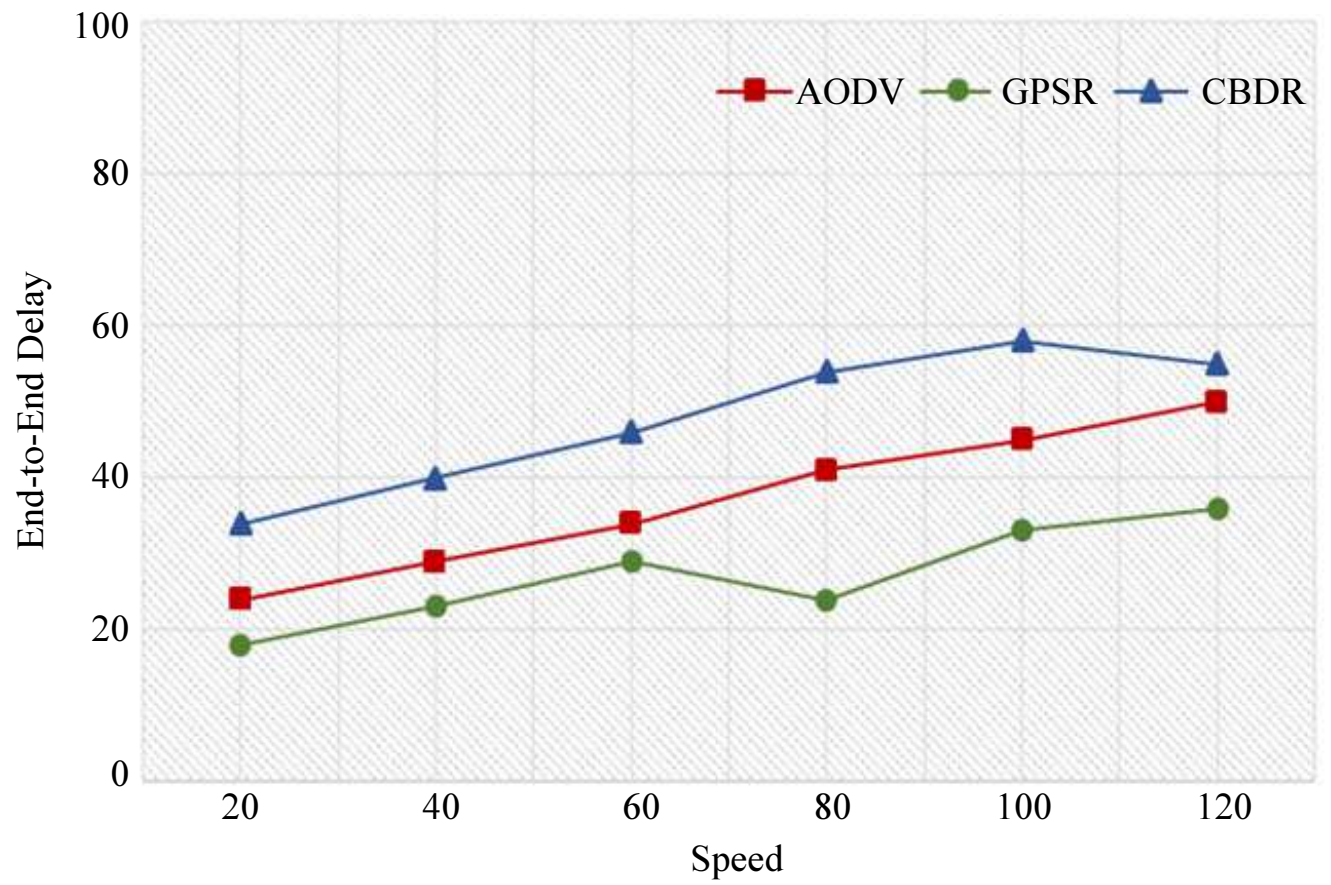

Fig. 11: Variation of End-to-End Delay over Speed in Gauss-Markov mobility model

\section{Conclusion}

Mobility models play an essential role in the precise simulation of the routing protocol in Vehicular Ad-hoc Networks. So, it is necessary to evaluate the performance of routing protocols on different models of mobility to see the impact of these models on different protocols.
In our studies, the performance of CBDR does not seem to be better. It provides a low packet delivery, low throughput and higher end-to-end delay. Contrary to GPSR that provide a good performance with the three mobility models. Also, using freeway mobility model gives a better evaluation of performance compared to other models, but this model is definitely 
unrealistic. Furthermore, the performance of routing protocols should be evaluated with the mobility model that most closely matches the expected real-world scenario. Actually, the anticipated real-world scenario can help the development of vehicular ad-hoc network protocols significantly. So, from the results we can say that Freeways is the better model for evaluating highway scenario and Street Random Waypoint is the best for urban scenario. In our future work we will propose an improved GPSR protocol and introduce its performance for urban and highway scenarios in real-world.

\section{Acknowledgment}

Authors would like to thank all the members of information and communication systems engineering research team for supporting this study.

\section{Author's Contributions}

Both authors were involved in implementation of the Routing protocols, analyzed the results, wrote the paper, review and correction of remarks.

\section{Ethics}

This article is original and contains unpublished material. The authors confirm that are no conflict of interest involved.

\section{References}

Alsaqour, R., M. Abdelhaq, R. Saeed, M. Uddin and O. Alsukour et al., 2015. Dynamic packet beaconing for GPSR mobile ad hoc position-based routing protocol using fuzzy logic. J. Netw. Comput. Applic., 47: 32-46. DOI: 10.1016/j.jnca.2014.08.008

Amici, R., M. Bonola, L. Bracciale, A. Rabuffi and P. Loreti et al., 2014. Performance assessment of an epidemic protocol in VANET using real traces. Proc. Comput. Sci., 40: 92-99.

DOI: $10.1016 /$ j.procs.2014.10.035

Divecha, B., A. Abraham, C. Grosan and S. Sanyal, 2007. Impact of node mobility on MANET routing protocols models. J. Digital Inform. Manage., 5: 19-24.

Houmer, M., M.L. Hasnaoui and A. Elfergougui, 2018. Security analysis of vehicular ad-hoc networks based on attack tree. Proceedings of the 7th International Conference on Selected Topics in Mobile and Wireless Networking, Jun. 20-22, IEEE Xplore Press, Morroco, pp: 21-26 DOI: 10.1109/MoWNet.2018.8428905

Kakarla, J., S. Sathya, G. Laxmi and R. Babu, 2011. A survey on routing protocols and its issues in VANET. Int. J. Comput. Applic., 28: 38-44.

DOI: $10.5120 / 3373-4663$
Karp, B. and H.T. Kung, 2000. GPSR: Greedy perimeter stateless routing for wireless networks. Proceedings of the 6th Annual International Conference on Mobile Computing and Networking, Boston, Aug. 6-11, pp: 243-254.

Katkar, P.S. and V.R. Ghorpade, 2016. Comparative study of network simulator: NS2 and NS3. Int. J. Adv. Res. Comput. Sci. Software Eng., 6: 608-612.

Khairnar, V.D. and K. Kotecha, 2013. Simulation-based performance evaluation of routing protocols in vehicular ad-hoc network. Int. J. Scientific Res. Public., 3: 1-14.

Kumar, R. and M. Dave, 2011. A comparative study of various routing protocols in VANET. Int. J. Comput. Sci. Issues, 8: 643-648.

Liang, B. and Z.J. Haas, 2002. Predictive distance-based mobility management for PCS networks. Proceedings of the Eighteenth Annual Joint Conference of the IEEE Computer and Communications Societies, Mar. 21-25, IEEE Xplore Press, USA, pp: 1377-1384. DOI: 10.1109/INFCOM.1999.752157

Martinez, F.J., J.C. Cano, C.T. Calafate and P. Manzoni, 2008. CityMob: A mobility model pattern generator for VANETs. Proceedings of the IEEE International Conference on Communications Workshops, May 19-21, IEEE Xplore Press, China, pp: 370-374. DOI: $10.1109 / \mathrm{ICCW} .2008 .76$

Mota, V.F.S., F.D. Cunha, D.F. Macedo, J.M.S. Nogueira and A.A.F. Loureiro, 2014. Protocols, mobility models and tools in opportunistic networks: A survey. Comput. Commun., 48: 5-19. DOI: 10.1016/j.comcom.2014.03.019

Poonia, R.C. and D. Bhargava, 2016. A review of coupling simulator for vehicular ad-hoc networks. Int. J. Inform. Technol. Comput. Sci., 5: 44-51. DOI: $10.5815 /$ ijitcs.2016.05.05

Rupinder, K. and S. Gurpreet, 2014. Survey of various mobility models in VANETs. Int. J. Eng. Comput. Sci., 3: 4073-4080.

Saini, H. and R. Mahapatra, 2014. Implementation and performance analysis of AODV routing protocol in VANETs. Int. J. Emerg. Sci. Eng., 2: 24-29.

Satyajeet, D., A.R. Deshmukh and S.S. Dorle, 2016. Heterogeneous approaches for cluster-based routing protocol in Vehicular Ad-hoc Network (VANET). Int. J. Comput. Applic., 134: 1-8. DOI: $10.5120 / \mathrm{ijca} 2016908080$

Singh, N., R.L. Dua and V. Mathur, 2012. Network simulator NS2-2.35. Int. J. Adv. Res. Comput. Sci. Software Eng., 2: 224-227.

Song, T., W. Xia, T. Song and L. Shen, 2010. A clusterbased directional routing protocol in VANET. Proceedings of the 12th International Conference on Communication Technology, Nov. 11-14, IEEE Xplore Press, China, pp: 1172-1175. DOI: $10.1109 /$ ICCT.2010.5689132 